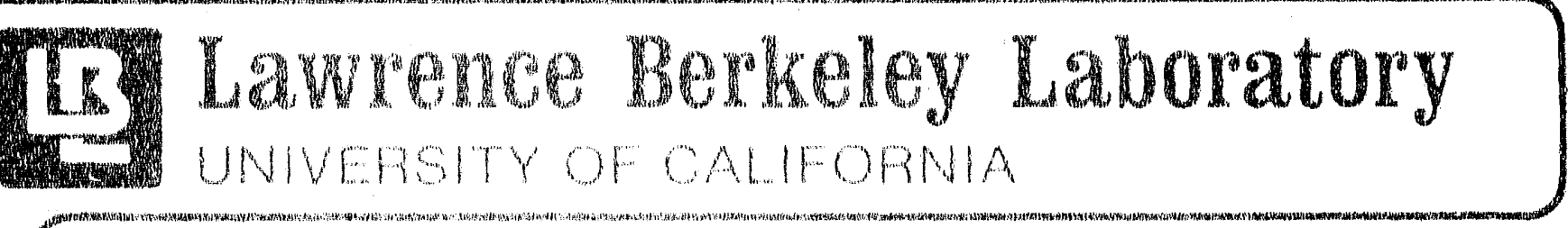

Presented at the Eighth Winter Workshop on Nuclear Dynamics, Jackson Hole, WY, January $18 \cdots 25,1992$, and to be published in the Proceedings

\title{
Thomas-Fermi Fission Barriers
}

W.D. Myers and W.J. Swiatecki

January 1992
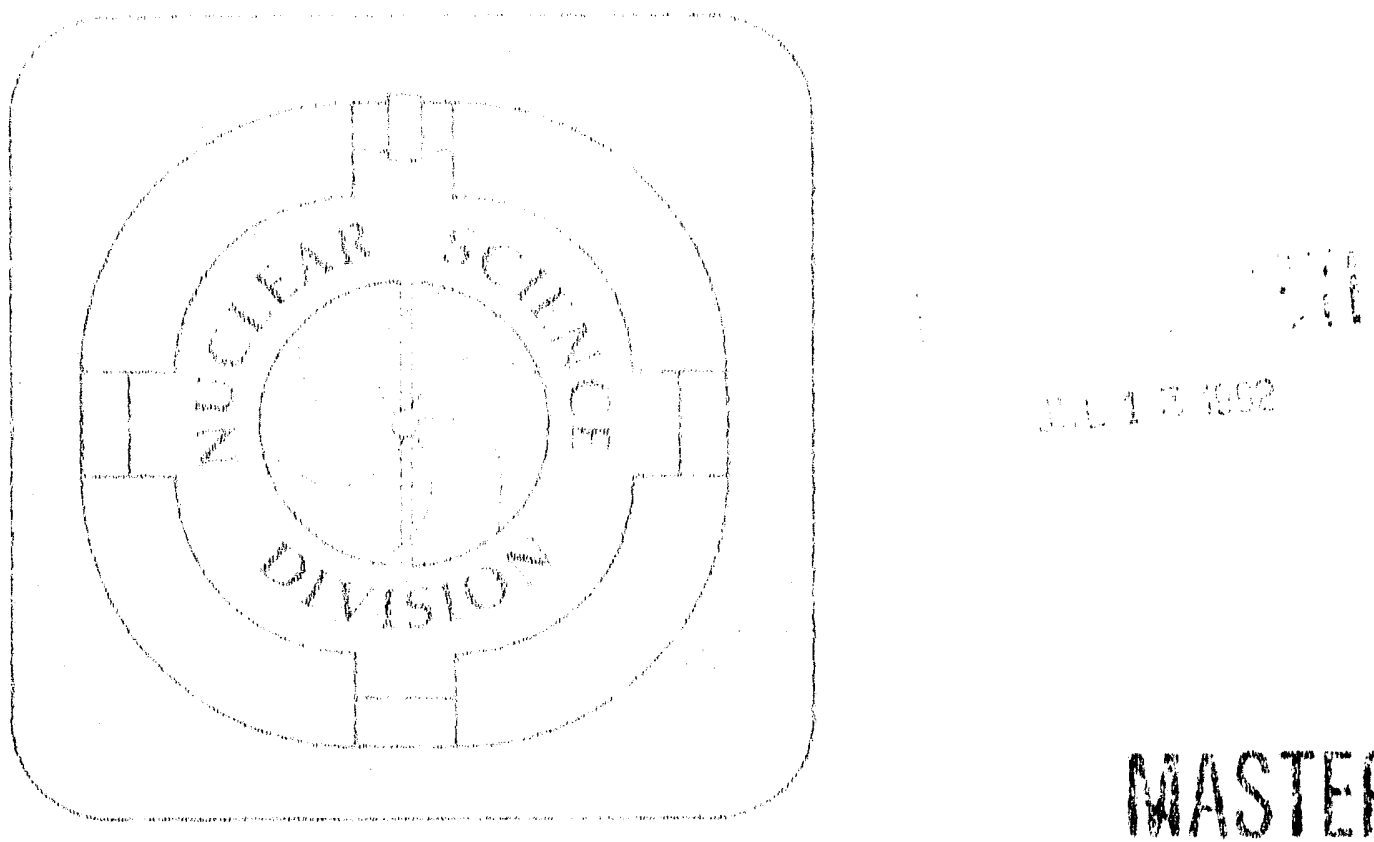

\section{MASTEP}

Prepared for the U.S. Department of Energy under Contract Number DE-AC03-76SF00098 DISTHISUTIOU OF THIS OOCHABNT IS IINLIMITEO 


\section{DISCLAIMER}

This dockment was proparid as an account of work sponsored by the Uniter States Government. Noither the United States Government nor any agency thered, nor the Regents of the Lisversity of Calfornia, nor any of their enployees, makes any wartanly, cxpress or im. plied, or assumes any legal liability or responsibitity for the accuracy, completeness, or usefulness of any information, apparatus, product, or process discloged, or represents that its use would not indringe privately owned rights. Roference herein to any specific commercial product, process, or service by its trade name, trademark, manufacfuret, or otherrise, does not necessarily constitute or imply its endorsement, recommendation, or favoring by the United States Govemment or any agency thereof, of The Regents of the University of Califomia. The views and opinions of authors expressed herein do not necessarily state or reflect those of the United States Covernment or any agency thereor or The Regerts of the University of California and shall not be usid for advertising or product endorsement purpixes.

Lawrence Berkeley Laboratory is an equal opportunity employer. 
$L B L-31908$

DE92 016992

\title{
THOMAS-FERMI FISSION BARRIERS *
}

\author{
WILLIAM D. MYERS and WLADYSLAW J. SWIATECKI \\ Nuclear Science Division \\ Lawrence Berkeley Laboratory \\ 1 Cyclotron Road \\ Berkeley, CA 94720, USA
}

\begin{abstract}
The Thomas-Fermi model of average nuclear properties described in Parts $I^{1}$ and $11^{2}$ is applied to the calculation of fission barriers and charge distributions. Comparison with experimental data reveals a barriers vs. size discrepancy. The suggestion is made that an extension of the Thomas-Fermi method is called for in order to describe the presence in nuclei of the "quantal halo," i.e. of the classically forbidden region around the nuclear surface where matter exists at negative kinetic energy.
\end{abstract}

\section{Introduction}

We have developed a model of average nuclear properties. It is based on a statistical treatment of the nuclear energy, analogous to the Thomas-Fermi approximation for the description of smoothed electron densities in atoms and molecules. In place of the electrostatic interactions between atomic electrons, an effective short-range nucleonnucleon potential was introduced, representing a generalization of the momenturndependent Seyler-Blanchard Yukawa interaction. The resulting model was used to discuss nuclear binding energies, sizes and charge distributions, as well as the relation of the nuclear compressibility to the surface energy and surface diffuseness. The nuclear optical model potential, including its energy and isospin dependenzes was also calculated. The interested reader should consult Parts $\mathrm{I}^{1}$ and $\mathrm{II}^{2}$ for a detailed description of this approach. (An extensive program of studies along similar lines is described in ref. ${ }^{3}$ or refs. ${ }^{4,5,6}$ and closely related work in refs. ${ }^{7,8}$ ) The present paper concerns only the confrontation of the model with measurements of nuclear charge distributions and fission barriers.

\section{Results}

The binding properties of nuclei were fitted ${ }^{9}$ (approximately) under the assumption that $r_{0}$ has the value $1.13,1.16$ or $1.19 \mathrm{fm}$. The resulting three sets of parameters

*This work was supported by the Director, Office of Energy Research, Office of High Energy and Nuclear Physics, Nuclear Physics Division of the US Department of Energy under Contract DE . AC03 - 76SF00098. 


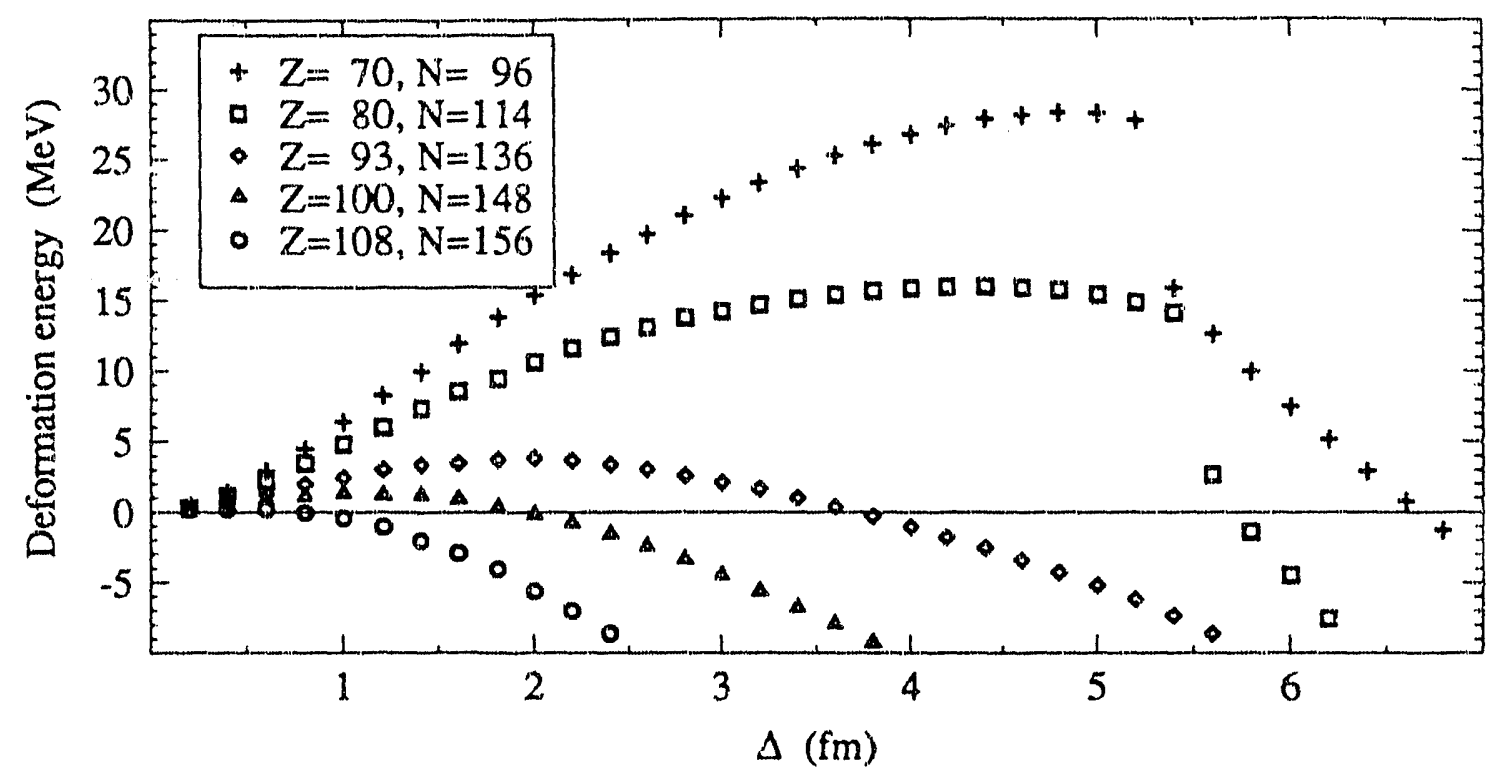

Fig. 1: Calculated deformation energies of conditional equilibrium shapes for five nuclei. The constraint parameter $\Delta$ is half the distance between the centers of mass of the two halves of the nuclear shape, less its value for the spherical configuration. The maximum in each curve corresponds to the fission barrier height. The break in the two upper curves corresponds to the point where the family of conditional equilibrium shapes comes to an end (by bending back at a limiting point). The continuation of this family (not shown) goes through another bend and re-emerges as the "fusion. valley" of two separated fragments with a constrained center-of-mass separation. Our iterative solu.tions of the Euler equations horne in on this valley and trace out its energy which, apart from some fragment deformation, corresponds simply to the Coulomb interaction between the fragments.

were used to confront the predictions of the present model with measured nuclear fission barriers and charge distributions.

The determination of the equilibrium configuration and energy of a nonspherical nucleus is difficult, since the Euler equations depend now on three position coordinates $x, y, z$. The technique we used for solving this problem numerically on a three-dimensional lattice is described in Part $\mathrm{II}^{2}$, Appendix $\mathrm{B}$.

Fig. 1 shows the result of fission barrier calculations for five nuclei, with atomic numbers $Z=70, \varepsilon 0,93,100$ and 108. The parameters corresponding to a radius constant of $r_{0}=1.16 \mathrm{fm}$ were used. The maximum of each curve corresponds to the height of the fission barrier for the given nucleus. Fig. 2 shows three barrier calculations for $Z=93, N=136$ using the parameter sets for $r_{0}=1.13,1.16,1.19$. Table 1 summarizes the calculated barrier heights and compares them with values deduced from experiment. 
By taking $r_{0}=1.16 \mathrm{fm}$ the calculated barriers for the heaviest elements can be made to agree with measurements. However, for $Z=80$ the calculated barrier is some $2 \mathrm{MeV}$ too high and for $Z=70$ the difference is about $4 \mathrm{MeV}$. As things stand, the fission barriers of the lighter elements cannot be reproduced even when the barriers for the heavy elements have been fitted (by adjusting $r_{0}$ to have the value $1.16 \mathrm{fm}$ ). In fact, however, the discrepancy is worse than that: the choice $r_{0}=1.16 \mathrm{fm}$ is unacceptable when the corresponding calculated charge distributions are compared with measurements.

Fig. 3 compares the measured charge distribution for ${ }^{124} \mathrm{Sn}$ with a ca culation in which the $r_{0}=1.16 \mathrm{fm}$ parameter set was used. The calculated curves have the charge distribution of the proton folded in, with an RMS radius of $0.85 \mathrm{fm}$ (and a Yukawa form factor). The pair of measured distributions displayed by solid lines represent parameterized two- and three-parameter fits to electron scattering data. It is clear that the calculated distribution is systematically too low in the bulk. Fig. 4 shows a similar comparison when the $r_{0}=1.13 \mathrm{fm}$ parameter set is used. The bulk density and radius are now in fair agreement, although the details (e.g. the fall-off profile in the surface) are still not reproduced. Taking the $r_{0}=1.13 \mathrm{fm}$ parameter set and recalculating the fission barriers, now leads to disaster: the barrier for the nucleus $Z=93, N=136$ comes out to be $8.3 \mathrm{MeV}$ instead of $3.8 \mathrm{MeV}$. Similarly for $Z=80, N=114$ we find $25.1 \mathrm{MeV}$ where measurements indicated $13.7 \mathrm{MeV}$. Thus we have a clear-cut discrepancy: using a Thomas-Fermi model adjusted to reproduce nuclear binding energies, a choice of $r_{0}$ equal to $1.13 \mathrm{fm}$ can reproduce nuclear sizes but not fission barriers. The choice $r_{0}=1.16 \mathrm{fm}$ would reproduce at least the barriers for heavy elements, but this value is simply not in agreement with measured sizes of nuclei.

Table 1. summarizes the calculated barrier heights and compares them with values deduced from experiment.

We have explored a number of additional parameter choices and hypotheses in order to test the firmness of the above conclusion, and we believe that, within the framework of the Thomas-Fermi approach, no adjustments of parameters can remove the discrepancy. It is true that, as regards nuclear radii, the discrepancy is no more than about $3 \%$, but the integrity of the model, the accuracy of the unparameterized solutions and the amount of data brought to bear on the determination of the model's parameters are such that we are inclined to consider this discrepancy as the signal of a relatively small but definite piece of physics that is missing in the ThomasFermi treatment of average nuclear properties. We regard this as one of the principal conclusions of this work.

The Thomas-Fermi treatment is a semi-classical approximation that works well in the bulk region of the nucleus but becomes less accurate in the surface and eventually fails completely in the sense that it allows no penetration of the particles into classically forbidden regions of space. Estimates show that even in heavy nuclei a significant fraction of the nuclear matter finds itseli in the classically forbidden re- 


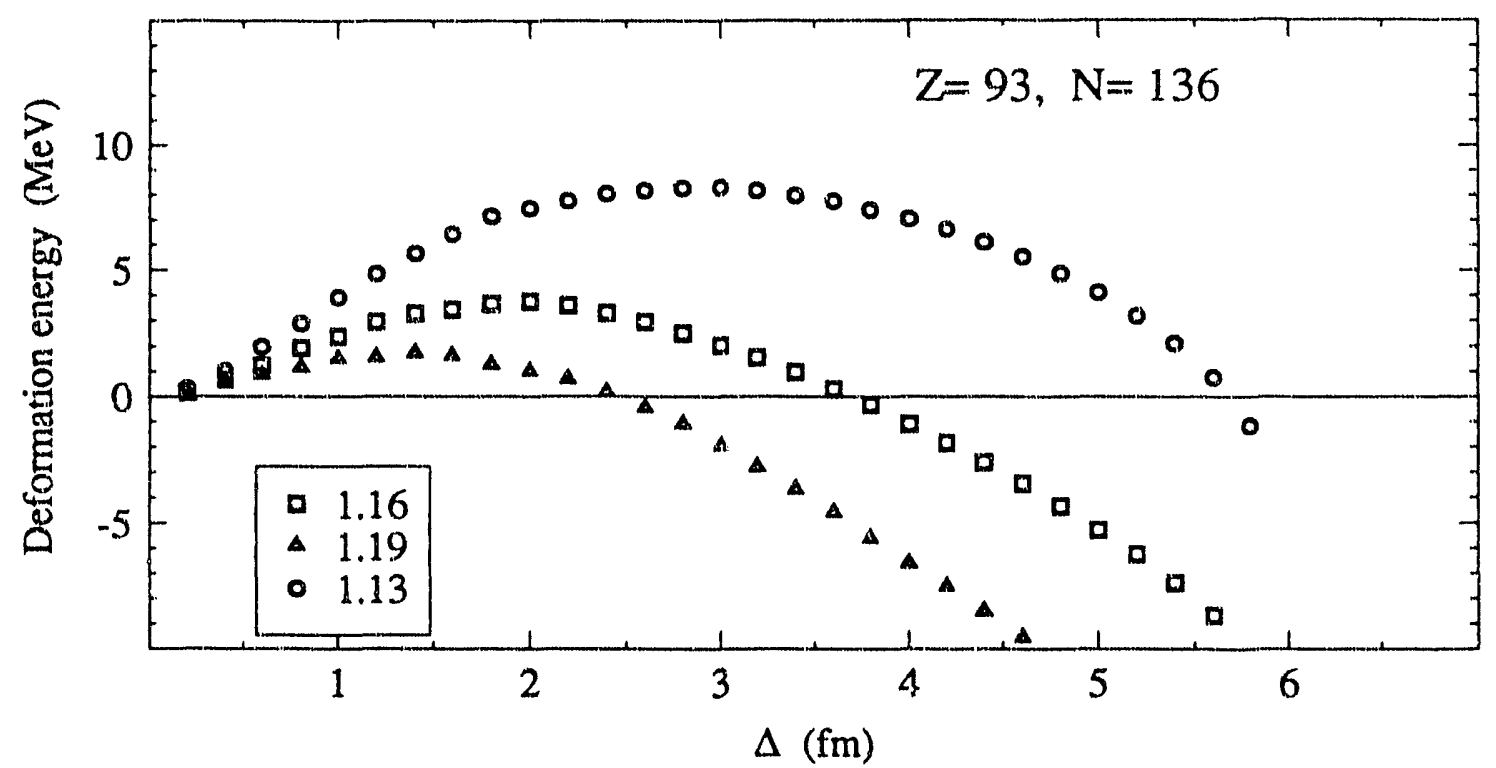

Fig. 2: This figure shows the fission barrier plots for the nucleus ${ }^{229} \mathrm{~Np}$. It illustrates the effect on the barrier height of changing the radius parameter $r_{0}$ while (approximately) preserving the fit to ground state masses.

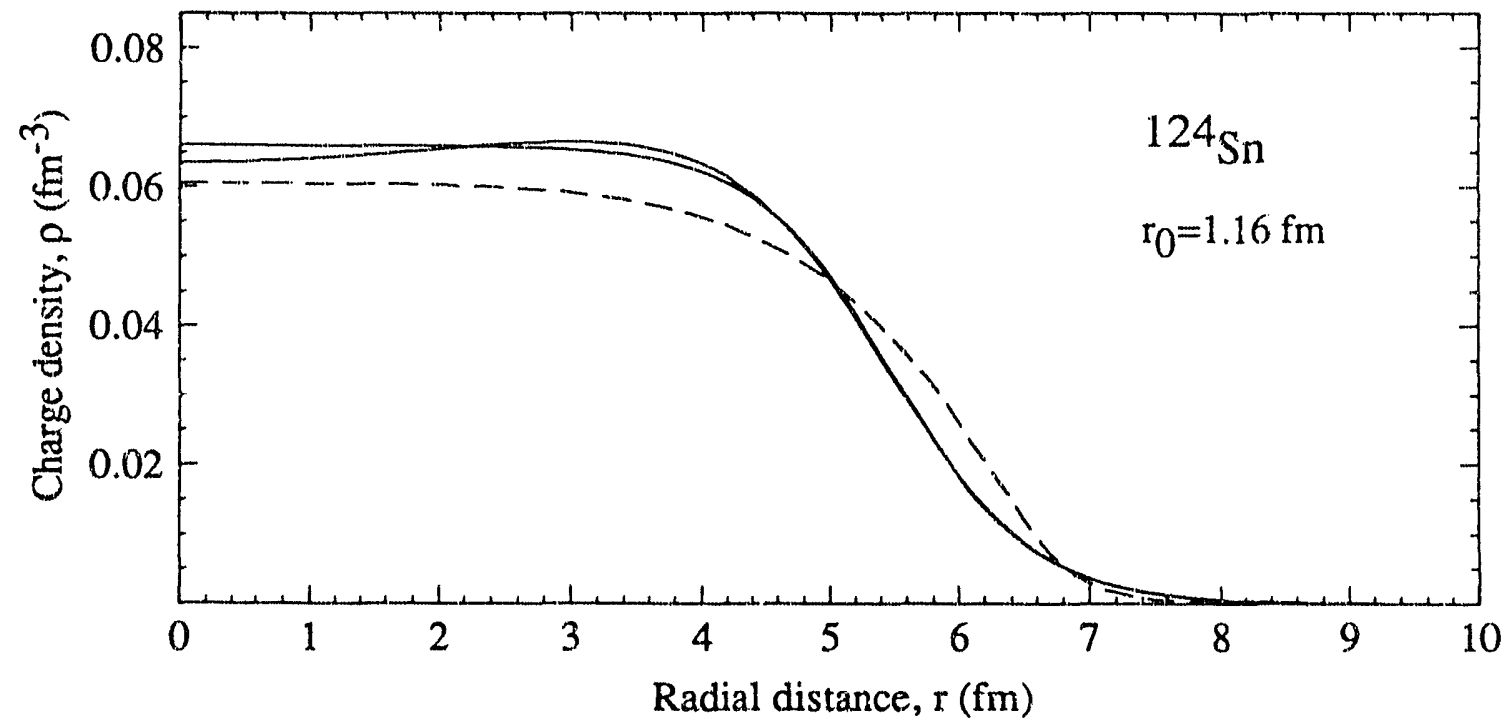

Fig. 3: The solid curves correspond to the measured charge distributions, as parameterized by a two-parameter Woods-îxon form factor, or a "three parameter Gaussian" form factor. The latter curves show a central depression in the charge distribution. The dashed curves correspond to the Thomas-Fermi calculations with the parameter set corresponding to $r_{0}=1.16$. A proton charge form factor (with a Yukawa shape and an R.MS size of $0.85 \mathrm{fm}$ ) has been folded in. 


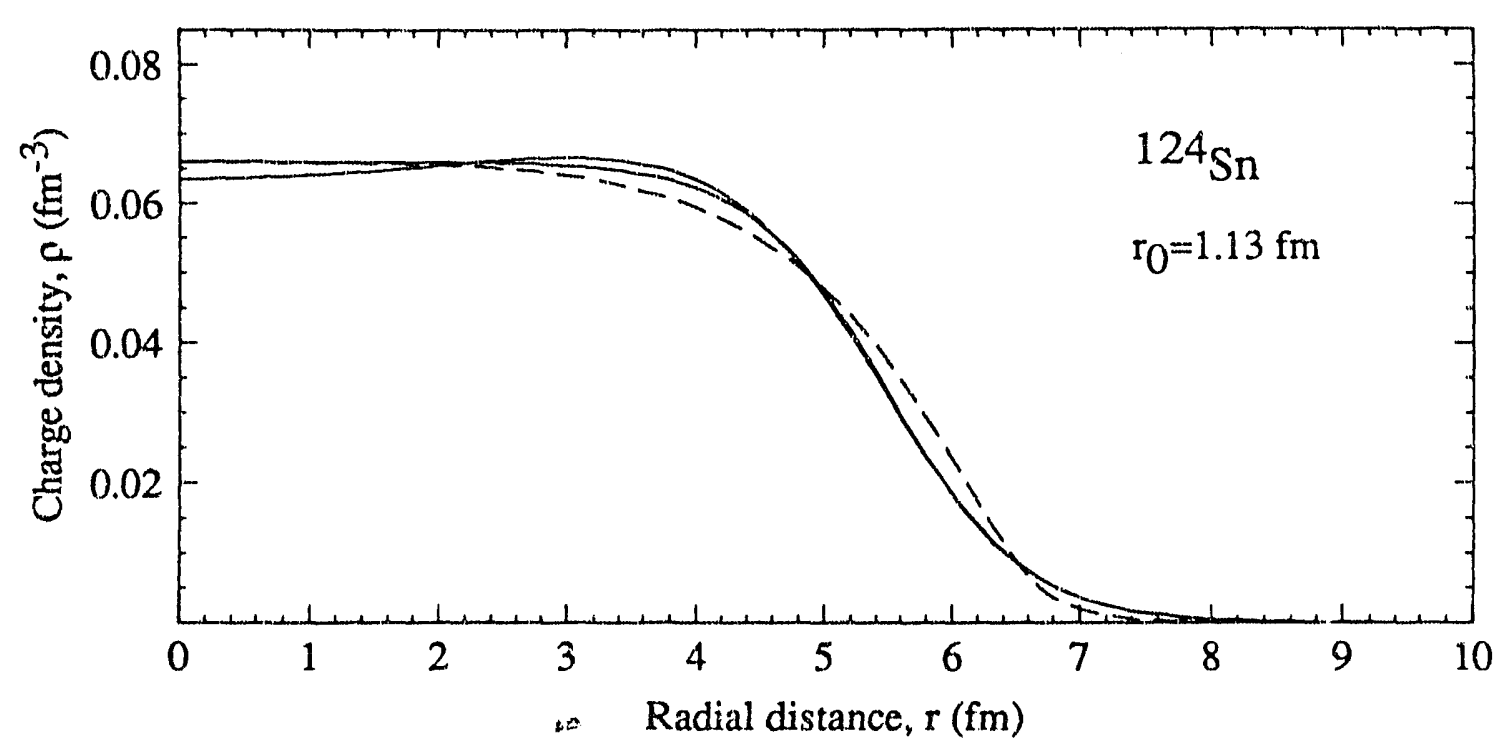

Fig. 4: This is like Fig.3, but with the parameter set corresponding to $r_{0}=1.13$.

Table 1: Experimental and calculated fission barriers.

\begin{tabular}{|c|c|c|c|c|c|}
\hline \multirow[b]{3}{*}{$\mathrm{Z}$} & \multirow[b]{3}{*}{$\mathrm{N}$} & \multirow[t]{3}{*}{ Experimental $(\mathrm{MeV})$} & \multicolumn{3}{|c|}{ Calculated $(\mathrm{MeV})$} \\
\hline & & & \multicolumn{3}{|c|}{ Value of the radius constant, $r_{0}(\mathrm{fm})$} \\
\hline & & & 1.13 & 1.16 & 1.19 \\
\hline 70 & 96 & $24.20^{6}$ & & $28.4(2.51)^{a}$ & \\
\hline 80 & 114 & $13.73^{b}$ & $25.1(2.58)$ & $16.0(2.64)$ & \\
\hline 93 & 136 & $3.78^{b}$ & $8.3(2.72)$ & $3.8(2.79)$ & $1.7(2.86)$ \\
\hline 100 & 148 & $1.5^{c}$ & & $1.3(2.87)$ & \\
\hline 108 & 156 & $0.3^{c}$ & & $0.2(2.93)$ & \\
\hline
\end{tabular}

${ }^{a}$ Numbers in parentheses refer to $\left\langle z>_{0}\right.$, where $2<z>_{0}$ is the distance in fermis between the centers of mass of the two halves of the spherical density distributions for the nucleus in question.

${ }^{6}$ Ref. ${ }^{10}$. Strictly speaking these are not experimental values, but values calculated by Sierk using a model ${ }^{11}$ which (when combined with shell effects) is known to give good agreement with experiment.

${ }^{c}$ Ref. ${ }^{12}$. 
gion, forming a quantal halo of material contributing positive density but negative kinetic energy density. One anticipated consequence of the presence of a fringe of negative kinetic energy in the outer layers of the surface is that such a surface should be relatively easier to bend, and the corresponding nucleus should be easier to deform. As a result, the calculated fission barriers should be lowered by the presence of the halo, possibly removing the above mentioned discrepancy. (For a discussion of a puzzle concerning the status of the curvature energy in nuclear problems, see ref. ${ }^{13}$ ) In a forthcoming paper we hope to examine the effect of the quantal halo on the size vs. barriers discrepancy uncovered in the present work. We are currently developing a generalization of the Thomas-Fermi model--simpler than conventional refinements ${ }^{4,14}$ - designed to accommodate the presence of matter possessing negative kinetic energy.

\section{Acknowledgements}

The authors wish to acknowledge discussions with J.R. Nix and A.J. Sierk and the substantial contributions that P. Möller made by determining the model parameters from a fit of the calculated binding energies to the measured masses of nuclei.

\section{References}

1. W.D. Myers and W.J. Swiatecki, Ann. Phys. (N.Y.) 204, 401 (1990).

2. W.D. Myers and W.J. Swiatecki, Ann. Phys. (N.Y.) 211, 292 (1991).

3. M. Brack, C. Guet and H.-B. Håkansson, Phys. Rep. 123, 275-364 (1985).

4. A. K. Dutta, J.-P. Arcoragi, J. M. Pearson, R. Behrman and F. Tondeur, Nucl. Phys. A458, 77-94 (1986).

5. F. Tondeur, A. K. Dutta, J. M. Pearson and R. Behrman, Nucl. Phys. A470, 93-106 (1987).

6. J. M. Pearson, Y. Aboussir, A. K. Dutta, R. C. Nayak, M. Farine, and F. Tondeur, preprint (1990).

7. F. Garcias, M. Barranco, J. Nemeth, C. Ngô, Phys. Lett. 206B, 177 (1988).

8. F. Garcias, M. Barranco, J. Nemeth, C. Ngô and X. Viñas, "Proc of the Symposium on Nuclear Dynamics and Nuclear Disassembly", Dallas, Texas (1989)

9. P. Möller, private communication, March 1990.

10. A. J. Sierk, private communication, April 1990.

11. A. J. Sierk, Phys. Rev. C33, 2039-53 (1986).

12. G. Münzenberg, Rep. Prog. Phys. 51, 57 (1988), Fig. 17.

13. W. Stocker, J. Bartel, J.R. Nix, A.J. Sierk, Nucl. Phys. A489, 252 (1988).

14. P. Ring and P. Schuck, "Nuclear Many Body Problem",Springer-Verlag, 1980. 
$\$$
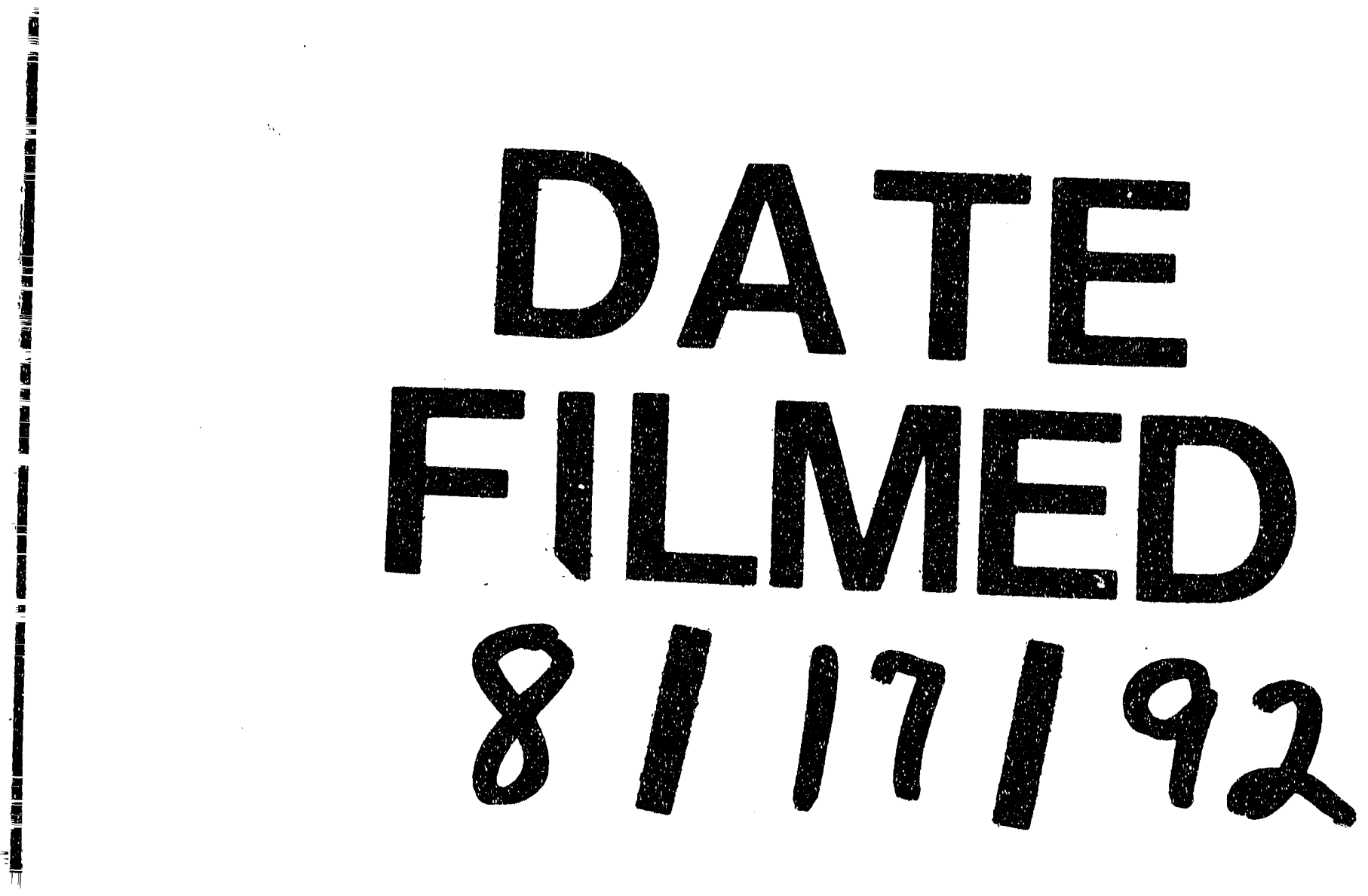
